\title{
Modelos de explicación, basados en prácticas experimentales. Aportes de la filosofía historicista
}

\author{
Explanation based models experimental practices. \\ Historicist philosophy contributions
}

\begin{abstract}
Resumen
En este documento se presentan aportes de la filosofía de las prácticas experimentales a la didáctica de la física. Inicialmente, se plantea el debate teoría-experimento y se hace énfasis en los aspectos fundamentales de la filosofía experimental; posteriormente se identifica la noción de "argumento experimental" para caracterizar la construcción del conocimiento científico; y, finalmente se establecen elementos de su influencia en la configuración de modelos explicativos en la enseñanza de la física.
\end{abstract}

\section{Palabras clave}

Filosofía de la ciencia, prácticas experimentales, didáctica de la física, modelos explicativos.

\section{Introducción}

La física está estructurada en dos grandes modalidades mutuamente relacionadas: la física teórica y la física experimental (Artigas, 1989). Por razones históricas, las reflexiones de la filosofía de la ciencia terminaron por favorecer el racionalismo y dar mayor peso a las teorías que a la experimentación. En vez de establecer un equilibrio entre ellas, terminó por colocar la una como subsidiaria de la otra. Los filósofos actuales reivindican el papel de la física experimental (Hacking, 1983) y establecen un giro en la reflexión filosófica, mostrando los aportes que la experimentación hace a la construcción del conocimiento científico mismo.

La didáctica de la física, que se nutre de los aportes de la filosofía de la ciencia, encuentra en este giro elementos de reflexión fundamentales en torno al papel del experimento; éste pasa de ser verificador o demostrador de teorías para convertirse en elemento de reflexión en sí mismo y de exploración de la riqueza conceptual que encierra.

* Profesor Asociado de la Universidad del Valle. Correo: edgarcia@univalle.edu.co 


\section{El debate teoría - experimento en la filoso- fía de la ciencia}

Tal vez resulta desapercibida para la didáctica de la física la controversia que existe en la relación teoría - experimento en los actuales debates de la filosofía de la ciencia. Para Iglesias (2004), estamos viviendo un cambio en la manera de concebir la relación entre ellas, desde el modelo establecido por la concepción heredada a una nueva perspectiva de la práctica experimental, basada en el principio de acción - intervención, y con ello en la transformación de la imagen de ciencia. Perspectiva que abre un espacio nuevo de posibilidades de exploración en la enseñanza de la física.

Realmente el debate sobre la relación teoría- práctica o sobre el papel de la teoría versus el experimento es antiguo en filosofía de la ciencia; lo relevante, que consideramos aquí, son los nuevos enfoques que surgen. Vamos a mostrar dichos enfoques, pero primero consideraremos la razón del cambio en la manera de percibir la naturaleza de la ciencia por parte de la filosofía de la ciencia.

\section{Los atisbos de la concepción heredada}

El éxito alcanzado por la física a finales del siglo XIX, particularmente la influencia del modelo newtoniano, y el lento desarraigo entre la tradición filosófica y la tradición científica, promovido por nuevos intereses, genera en la naciente filosofía de la ciencia la necesidad de establecer criterios de demarcación, provenientes del método de la física. También surge el requerimiento de centrar el interés en el cómo hacer dentro de otras disciplinas (sociales y humanas), lo que los físicos y químicos han hecho de manera tan exitosa. Esto es, ¿cuáles son las reglas que determinan el buen método de investigación científica? (Guerrero, 2006). El fin de diferenciar la ciencia de actividades pseudo científicas hace necesario crear una demarcación entre ciencia y pseudociencia (metafísica); esto es, entre la racionalidad científica y otras formas de opinión, tales como la religión, el arte y la ética. La preocupación por el método de la ciencia se convirtió en una razón poderosa para la naciente filosofía de la ciencia. Los llamados filósofos del método como los describe Pérez Soto (1998) se preocuparon por estudiar la estructura de las teorías científicas, siendo un gran referente el modelo newtoniano. Los criterios, un poco restringidos aquí, tenían que ver con aspectos relacionados con racionalidad, verdad, objetividad y mundo.

Al Círculo de Viena se le reconoce una enorme influencia en esta dirección. Los trabajos de Carnap, Schilck y Hanson son representativos de una corriente de pensamiento reconocida como positivismo o empirismo lógico, caracterizada posteriormente como la concepción heredada. Dentro de las múltiples preocupaciones del positivismo en torno a la naturaleza de la ciencia, nos ubicaremos en este documento solamente en una, la que tiene que ver con la relación teoría - práctica.

La preocupación de los positivistas sobre la naturaleza del conocimiento estuvo orientada fundamentalmente al estudio de las teorías científicas; en los modelos teóricos, las leyes y principios establecidos. De hecho, una de las tareas de la filosofía de la ciencia ha sido el análisis y reconstrucción de las teorías científicas: ¿Qué ha de entenderse por evidencia científica? ¿Qué se entiende por teoría científica? ¿En qué consiste el progreso científico? ¿Cuál es la función y características de la explicación científica? El debate sobre la dependencia/ independencia teórica de la observación y el problema de la evaluación de las teorías científicas (Guerrero 2006) han sido preguntas muy recurrentes. Incluso, el estudio de las propias disciplinas, como el caso de Frege en Aritmética, Russell en Física, Hilbert en Geometría... La búsqueda, entre otros asuntos, de poder brindarle el estatus adecuado al razonamiento lógico del pensamiento científico.

Para algunos filósofos actuales, como Hacking (1983) y Estany (2007), un aspecto relevante de la concepción heredada fue el papel subsidiario que le correspondió al experimento. El problema de la verificación estaba ligado al de la predicción de las teorías, como lo sugiere Popper cuando afirma que "Sólo cabe realizar experimentos a la luz de las preguntas y los conceptos determinados por una teoría" (1935). 
En un principio, verificar era poder constatar los enunciados en su sentido lógico, se creía en la posibilidad de una verificación concluyente de los enunciados científicos a partir de proposiciones elementales. Posteriormente, se fue derivando hacia tesis menos estrictas, llegando a considerar, como lo hace Schlick (1979), que el último paso de verificación ha de consistir en observaciones o en percepciones de los sentidos. Lo esencial del saber científico, para el Círculo de Viena, es la capacidad de predecir exactamente fenómenos físico-naturales (Echeverria, 2003). El demostrar o verificar lo que predice una ley o una teoría era razón fundamental para considerar su validez científica. La influencia de Carnap y Hempel ayudó para que las ideas de verificación asociadas a las teorías científicas fueran consideradas casi como la única forma legítima de proceder científicamente. Para esta concepción de ciencia, el experimento es considerado como verificador de teorías, tal como lo consideró el empirismo lógico (o falseador de las mismas desde un punto de vista popperiano).

El papel subsidiario de la experimentación ha sido entonces una característica de la tradición heredada, pero su influencia se mantuvo incluso en visiones como la de Kunh, que a pesar de romper con la doctrina positivista, conserva la tendencia a dar el peso a los modelos teóricos sobre la experimentación (Iglesias, 2004). Para Hacking (1983), la experimentación ha estado en función de la teoría, ya sea inspirada por ella o al servicio de la misma, pero en cualquier caso, sin vida propia.

\section{El giro hacia la filosofía de las prácticas experimentales}

El "reclamo" a la filosofía de la ciencia está en llamar la atención sobre la importancia de la experimentación en la construcción misma del conocimiento científico, y no como agregado subsidiario de los modelos teóricos. La manera de presentar los experimentos no debe ser solamente descriptiva o narrativa para reforzar el papel de las teorías, sino que debe avanzar hacia la caracterización de experimentos que involucren problemáticas y que tengan una riqueza conceptual en sí mismos, o como dice Hacking (1983), "que la experimen- tación tenga vida propia”. Esto es, crear necesidades donde el experimento "hable" y se comunique, crear situaciones específicas donde la naturaleza se "despliegue" y muestre comportamientos.

Gran parte de este "reclamo" se fundamenta en estudios de caso, donde filósofos historicistas como Hacking (1983), Galison (1987), Pickering (1995), Heilbronn (1979), Gooding, Pinch y Schafer (1989), Steinle (2002), Franklin (2002) y Martínez (2003) entre otros, muestran con evidencias el papel que ha jugado la experimentación en la construcción del conocimiento científico. Por ejemplo, Heilbronn (1979) en sus análisis históricos sobre electricidad afirma que este juego exploratorio posibilitó la distinción entre materiales conductores y no conductores. En los estudios de Steinle (2002), la simple variación de lugar de la aguja respecto al hilo en el experimento de Oersted, planteaba dificultades al enfoque de Newton-Laplace de fuerzas centrales. El resultado de estos trabajos pone en evidencia que no necesariamente la experimentación debe tener un papel subsidiario. En palabras de la Iglesias:

Cuando se asume la perspectiva de estudios de casos, se encuentra que no es la teoría la que siempre ha guiado la ciencia, que la relación entre teoría y experimento ha sido diversa y no unitaria como han querido mostrarnos la historia y la filosofía de la ciencia (2004).

Esto pone en evidencia que la miseria del teoreticismo (como la denominan algunos filósofos) está en reducir la riqueza y la complejidad del proceder científico a un asunto de mera elaboración conceptual, dejando de lado la riqueza de conocimiento que se esconde detrás de las prácticas experimentales. El giro hacia la práctica en filosofía de la ciencia obliga a que los temas de racionalidad, objetividad, verdad y mundo dejen de ser tratados desde la teoría, y a que se redefinan nuevos problemas filosóficos, promoviendo así una nueva imagen de la ciencia (Iglesias, 2004).

En la propia experimentación es importante distinguir entre experimentos cualitativos y cuantitativos o experimentación exploratoria y experimentación guiada. La experimentación cualitativa, que suele 
estar presente en las primeras fases del desarrollo de una ciencia, no es menos valiosa que los experimentos cruciales (experimentación cuantitativa guiada) en la estructuración de la ciencia; este tipo de experimentación resulta interesante desde el punto de vista epistemológico porque permite caracterizar la edad temprana de una ciencia. Para la escuela positivista, los experimentos cuantitativos eran el referente de las investigaciones para describir los procesos de elaboración de teorías científicas a partir de mediciones y datos cuantitativos precisos; fue esta versión simplificada del método científico la que llevó a borrar del mapa la experimentación cualitativa. Al respecto Ordóñez y Ferreirós sostienen que:

\begin{abstract}
Al menos en física, los experimentos cualitativos han sido una parte fundamental de los procesos de formación de conceptos (procesos de formación de datos). Por ejemplo, los experimentos cualitativos en electromagnetismo desempeñaron, desde el primer resultado de Oersted en 1820, un papel fundamental en la elaboración de nociones como líneas de fuerza y campo. Oersted y el propio Faraday trabajaron de manera más intuitiva y directa, modelando sus concepciones según algunos rasgos fenomenológicos (o fenomenotécnicos) que surgían directamente de los experimentos que realizaron y como resultado del experimento, el modelo fenoménico es refinado, acomodado y especificado con mayor precisión. (2002)
\end{abstract}

El caso de Faraday es llamativo porque los filósofos historicistas han encontrado que sus trabajos fueron una genuina base experimental; los experimentos eran creados, los aparatos se diseñaban y se construían para producir y evidenciar efectos deseados. También, implica tomar en cuenta otros factores que intervienen en el quehacer científico, como el anclaje material, la interacción humana, las relaciones con las administraciones, etc. Aunque no son totalmente independientes estos factores, inciden de forma distinta y en mayor o menor grado en la dinámica interna de la ciencia, lo que hoy en día hace referencia a la fenomenotecnia. (Estany, 2007). Por tanto, el debate que se ha planteado en las últimas décadas en filosofía e historia de la ciencia no es en torno a si los científicos han realizado experimentos en sus investigaciones, sino más bien en la interpretación de los mismos y en su relación con los modelos teóricos.
Para establecer el equilibrio necesario, Estany (2007) sugiere una interacción entre ambas, teoría y experimentación, donde la una no sea subsidiaria de la otra sino que se complementen mutuamente. Para ello, es necesario replantear las formas de representar el conocimiento, donde la denominación de "práctica científica" (que hace referencia a la actividad de los científicos) no esté determinada sólo por el producto (teorías) sino también por el proceso de dicha actividad. Un aporte en esta dirección lo sugiere Hutchins (1995) al proponer el enfoque de la cognición socialmente distribuida, en el que la unidad de cognición no es la mente individual sino un sistema formado por la interacción entre diversos agentes, y entre éstos y determinados artefactos tecnológicos implicados en el éxito del proceso cognitivo. Finalmente, debemos reconocer que la nueva imagen de ciencia está orientada por una relación entre la teoría y el experimento mucho más profunda y con mayor riqueza conceptual de la que la mayoría de los filósofos e historiadores han mantenido hasta el momento.

Reconocer, entonces, la importancia y la validez de las prácticas experimentales en la constitución de la ciencia, su función independiente de la teoría o en equilibrio con ella, y su papel más allá del verificacionista o falsacionista que usualmente se le ha otorgado, constituye el fundamento de este campo de investigación de la filosofía de la ciencia.

\section{El argumento experimental}

Otro aspecto que resulta importante desde la filosofía de la experimentación es el tipo de discurso que hay en la propia experimentación. Es un discurso que no se corresponde con el que en la concepción heredada se le asigna a la inducción y la deducción. En la experimentación existe una forma de argumentación y de conocimiento diferente al fenómeno de la deducción (Galison 1987). Es necesario admitir que en la acción hay pensamiento, hay un lenguaje diferente que se expresa en la actividad experimental y del cual surgen pensamientos e ideas que posteriormente se articulan conceptualmente. El conocimiento experimental está presente al diseñar y construir aparatos, también lo es manipular entidades y crear fenómenos. 
Para esta línea de pensamiento resultan interesantes las aportaciones de Pickering (1995) sobre tres aspectos que se encuentran en la producción de cualquier resultado experimental. Veámoslos a través de un sencillo ejemplo tomado de la historia de la física, ellos son:

1. Un procedimiento material: supone disponer de los aparatos e instrumentos necesarios, verificar que funcionen y controlar su funcionamiento, todo lo cual encierra un conocimiento práctico.

El conocimiento práctico que encerró en la Edad Media el diseño y la construcción de la máquina de vacío o bomba neumática, como se le conoce ahora, jugó un papel relevante en el siglo XVII para Robert Boyle y su ayudante Robert Hook. La inquietud fundamental era ¿cómo extraer el aire completamente de la campana? Para resolver este problema se diseñó un sistema de cremallera con una manivela que se moviera un pistón hacia arriba y hacia abajo, para poder controlar la salida del aire con una válvula. El artefacto funcionó perfectamente, pero lo extraño era que no lograba sacar totalmente el aire de la campana. Las dificultades técnicas del montaje, evitar que el aire se escapara por los poros de las tapas herméticas, controlar la observación al interior de la máquina le implicó a Boyle un conocimiento práctico. Ensayó con cremalleras más resistentes, campanas más pequeñas y materiales distintos, pero no pudo resolver este problema técnico. Preocupado por el funcionamiento de la máquina llegó a pensar que tal vez el problema no fuera de la máquina misma sino del comportamiento del aire al interior de la campana, idea que lo guió posteriormente a proponer el carácter elástico del aire y el modelo de resortes.

2. Un modelo instrumental: está implicado en el diseño, realización e interpretación del experimento, siendo fundamental la comprensión conceptual del funcionamiento de aparatos e instrumentos.

El funcionamiento de un aparato debe estar en relación con la intencionalidad que lo motiva.
La intención de Boyle era replicar el célebre experimento de Torricelli al interior de la máquina y verificar la hipótesis de que "es el peso de la columna de aire exterior la causa de que la columna de mercurio no caiga del tubo". Al realizar la experiencia se dio cuenta que la columna de mercurio no caía totalmente, como debía suceder. Había dos interpretaciones posibles: el experimento estaba mal diseñado o la máquina no funcionaba correctamente. Como la causa finalmente fue atribuida al comportamiento del aire al interior de la máquina, entonces Boyle diseñó otros experimentos utilizando tubos en $U$ para verificar el comportamiento del aire, llegando a la conclusión que hay una relación inversa entre la presión del aire encerrado y el volumen que ocupa.

3. Un modelo fenoménico: consiste en la comprensión conceptual de los aspectos del mundo fenomenológico, que están siendo estudiados por parte del experimentador, y sin los cuales los resultados carecerían de sentido y significación, por lo que no podrían ser interpretados.

Desde los griegos se consideraba, por razones teológicas, que el vacío era imposible en la Naturaleza. La autoridad de los argumentos de Aristóteles fue suficiente para que los escolásticos defendieran esta idea durante muchos siglos. El experimento de Torricelli se hizo "famoso" por que sugería la existencia del vacío e iba en contra de las doctrinas establecidas. Boyle, por una parte comparte la tesis de Torricelli y por otra se identifica con la experimentación realizada por Pascal sobre el peso del aire, por lo que ahora quiere demostrar la existencia e inactividad del vacío y argumentar a favor de la acción del peso del aire, utilizando la máquina neumática.

¿Por qué Boyle termina atribuyendo un comportamiento elástico al aire? El estudio de los resortes se había convertido en algo relevante para la mecánica y Hooke aportó en esta dirección. Para Boyle, la resistencia del aire a salir de la campana era similar a la resistencia de un resorte a ser estirado, entonces por analogía era necesario identificar la 
constante de elasticidad que hay en el aire encerrado. Boyle lo logró en un experimento con un tubo en $\mathrm{U}$ en el que controla dos variables, la cantidad de aire encerrado (volumen) y la presión que podía soportar (peso de la columna de mercurio). Posteriormente fue reconocida como la ley de Boyle.

En esta manera de caracterizar Pickering, la experimentación nos permite identificar aspectos relevantes de la experimentación en la construcción del conocimiento que no suelen ser reconocidos por historiadores y filósofos de la ciencia positivista y cuya influencia es cada vez mayor en la enseñanza de la física.

\section{Modelos explicativos y enseñanza de la física}

Un aspecto que se ha considerado en las investigaciones actuales en enseñanza de la física es la noción de modelo o "modelizar". Existen interpretaciones diferentes y aplicaciones distintas sobre lo que ello significa; se suele hacer referencia a modelos didácticos, modelos físicos, modelos análogos, modelos teóricos, modelos de ciencia..., pero la noción de modelo es diferente. Para nuestro caso, asumimos un "modelo" de acuerdo con la propuesta que hace R. Giere, como formas de caracterizar y organizar los hechos y fenómenos objetos de estudio. Son construcciones humanas y por tanto solamente representan una perspectiva de la realidad, más no la realidad misma. Para R. Giere (1999), la relación entre modelo-realidad "no es global, sino solo relativo a aquellos aspectos del mundo que se quieren capturar". Pueden existir familias de modelos agrupados de acuerdo a la intencionalidad que guía la interacción con la realidad; incluso, puede asumirse que el núcleo del conocimiento lo constituyen el conjunto de modelos que la estructuran. Desde esta perspectiva los modelos se generan para dar respuesta a una manera de observar la realidad (Guidoni, 1990), lo cual posibilita establecer formas de preguntarse por ella y hacer predicciones que se contestan en la interacción. En este sentido, la realidad observada forma parte del modelo porque se genera en relación a ella y porque ella se observa a través de él (Sanmartí 2002). Una manera de construir y organizar modelos es a través de las prácticas experimentales.

En los procesos de enseñanza de las ciencias, la experimentación suele estar presente: los estudiantes hacen prácticas, observan fenómenos, toman datos, hacen registros y a veces manipulan aparatos. Los libros de texto sugieren en muchos casos la realización de tales experimentos; pero cabe preguntarnos: ¿Cuál es la finalidad de los experimentos sugeridos? ¿Qué modelos se promueve desde las prácticas sugeridas? ¿Qué intención orienta al autor cuando presenta experimentos? (García \& Estany 2010). Una revisión del tema nos lleva a considerar la poca importancia dada a la experimentación en los libros de texto y, menos aún, a la experimentación cualitativa. La finalidad de los experimentos sugeridos en los textos es fundamentalmente demostrativa, esto es, validar o confirmar lo que dice la teoría (García, 2011). En un sencillo ejemplo de física podemos notarlo. En el texto de física general de Paúl Tipler encontramos la siguiente afirmación:

La conservación de la carga puede ilustrarse mediante un método simple de cargar un conductor llamado carga por inducción. Dos esferas metálicas sin carga están en contacto. Al acercar a una de las esferas una barra cargada, los electrones fluyen de una esfera a la otra, acercándose a la barra si esta se encuentra positivamente cargada o alejándose si su carga es negativa. Si los conductores se separan en cargas iguales y opuestas se dice que está polarizado. (Tipler, 2005)

Esta manera de presentar el tema por parte del libro de texto, muestra la influencia de la visión teorética y el carácter subsidiario del experimento. No hace falta hacer el experimento para entenderlo, y si se hace es para verificar el enunciado. En los cursos de física donde existen prácticas de laboratorio, este modelo suele ser considerado a tal punto que se presenta en pasos o secuencias que se deben dar para obtener el resultado esperado, como una receta de cocina. Este modelo explicativo no se corresponde con la propuesta de Giere.

La historia de la física, por el contrario, es rica en experimentación y aporta significativamente en esta dirección. Considerar estos aspectos en la enseñanza de la física a través de la recontextualiza- 
ción de saberes permite avanzar en la construcción significativa del conocimiento.

Como alternativa para ser tenida en cuenta en el diseño de unidades didácticas, basadas en las prácticas experimentales se sugiere tener en cuenta los siguientes aspectos:

a) Experimentos cualitativos: no solamente para describirlos, sino para mostrar su relevancia en la construcción de conocimiento. En este sentido la historia de la física es rica y variada para seleccionar este tipo de experimentos y encontrar la riqueza conceptual que poseen. Construir modelos explicativos en torno a la experimentación cualitativa es pues una necesidad en la comprensión fenoménica de la física.

b) La creación de artefactos y el procedimiento material: el conocimiento práctico que se encuentra en el diseño, creación, construcción $y$ funcionamiento de aparatos e instrumentos son relevantes en la construcción de conocimiento. No se trata necesariamente de hacer réplicas iguales a los originales, es mas bien la comprensión conceptual que encierra. Construir modelos explicativos sobre el diseño y funcionamiento de aparatos e instrumentos de medida permite comprender los fenómenos de manera significativa

c) Contextualizar la experimentación: la riqueza conceptual que no se ha considerado en la experimentación, al mantener el papel subsidiario, se hace ahora significativa. Las problemáticas, dificultades, necesidades e intereses de los científicos se hacen relevantes, en tanto que permite considerar los aspectos del mundo que están en juego: las problemáticas sociales e ideológicas, los conflictos entre teorías o visiones de mundo y el sentido mismo de los experimentos cruciales.

Cada uno de estos aspectos aporta elementos para la construcción de modelos de explicación de los fenómenos físicos, modelos que se contrastan y enriquecen, modelos que se amplían y reorganizan, modelos dialógicos, en fin, modelos que permitan intervenir y transformar el mundo en el que se vive.

\section{A manera de conclusión}

Es posible entonces reorientar el papel que se le está dando a la experimentación y considerar la riqueza conceptual que allí se esconde. Saber física no es solamente saber de leyes y teorías, es necesariamente la interacción con el saber experimental, es darle importancia al análisis del diseño y construcción de aparatos e instrumentos, de experimentos cualitativos y cuantitativos cuya riqueza esté en llenar de sentido el conocimiento.

Es importante que los libros de texto, que constituyen una forma de anclar materialmente los conocimientos, no presenten la experimentación como subsidiario de la teoría; sino mas bien, que contribuyan al equilibrio y complementariedad entre teoría y práctica. En este sentido, es necesario avanzar en propuestas de diseño de libros de texto que le den un giro a la experimentación en los términos planteados por la filosofía de las prácticas experimentales ${ }^{2}$.

Finalmente, se ha querido poner en evidencia la fortaleza de la práctica científica, donde se abre un panorama de posibilidades de exploración experimental y conceptual en la que aparatos e instrumentos se hacen relevantes en la construcción del conocimiento. La experimentación se establece así como una forma genuina de organizar fenómenos, donde el estudiante puede asumir un papel más activo en la construcción de explicaciones que le permitan hacer comprensible y transformable el mundo que le rodea.

\section{Bibliografía}

Artigas, M. (1989). Filosofía de la ciencia experimental; Introducción. España: Ediciones Universidad de Navarra.

Echevarria, J. (2003). Introducción a la metodología de la ciencia. La filosofía en el siglo XX. Madrid: Cátedra.

2 De hecho algunas propuestas se vienen realizando. Véase por ejemplo el libro Historia de la Ciencia en Textos para la Enseñanza. ed. Universidad del Valle. (García 2009) 
Estany, A. (2007). "Innovación tecnológica y tradiciones experimentales. Una perspectiva cognitiva". Revista ciencias. Facultad de ciencias. UNAM. México.

Galison, P. (1987). How experiments end. University of Chicago Press. Chicago.

García, E (2009). Historia de la ciencia en Textos para la Enseñanza. Cali: Editorial Universidad del Valle.

García, E. (2011). Las prácticas experimentales en los textos y su influencia en el aprendizaje. Tesis doctoral. Barcelona: Universidad Autónoma de Barcelona.

García, E \& Estany, A. (2010). "Filosofía de las prácticas experimentales y enseñanza de las ciencias". Revista Praxis Filosófica, núm. 31. Departamento de Filosofía. Cali: Universidad del valle.

Giere, R. (1990). Explaining science: a cognitive approach. Chicago: The University of Chicago Press, XXI. Chicago.

Gooding, D., Pinch, T. \& Schaffer, S. (1989). The uses of experiment. Cambridge: University Press.

Guerrero, G. (2006). Introducción a la filosofía de la ciencia. Documentos de trabajo. Cali: Universidad del Valle.

Guidoni, P., Arca, M. \& Mazzoli, P. (1990). Enseñar ciencia. Como empezar: reflexiones para una educación científica de base. Barcelona: Paidós.

Hacking, I. (1983). Representing and Intervening: Introductory Topics in the Philosophy of Natural Science. Cambridge: Cambridge University Press.
Hacking, I. (1996) Representar e Intervenir. Trad. Sergio F. Martínez, Instituto de Investigaciones Filosóficas - UNAM. México: Paidós.

Heilbron, J. (1979). Electricity in the $17^{\text {th }}$ and $18^{\text {th }}$ centuries. California: University of California Press. Berkeley.

Hutchins, E. (1995). Cognition in the wild. The MIT Pres.

Hutchins, E. (2005). "Material anchors for conceptual blends". Journal of pragmatics, núm. 37.

Iglesias, M. (2004). "El giro hacia la práctica en filosofía de la ciencia: una nueva perspectiva de la actividad experimental". En Revista de ciencias humanas y sociales, núm. 20, pp 44.

Kuhn, T. (1962). La estructura de las revoluciones cientificas. México: FCE.

Martinez, S. M. (2003). Geografía de las prácticas científicas. México: UNAM.

Ordoñez, J. \& Ferreiros, J. (2002) "Hacia una filosofía de la experimentación”. En Crítica, revista hispanoamericana de filosofía, vol. 34, núm. 102. pp.47-86.

Pickering, A. (1995). The mangle of practice. Time, agency and science. The University of Chicago Press.

Popper, K, R. (1935). La lógica de la investigación científica. (Trad.) Víctor Sánchez de Zavala. 1962. Madrid: Tecnos.

Steinle, F. (2002). "Challenging established concepts. Ampere and exploratory experimentation". En Teoría, vol. 17, núm. 44. pp. 291-316.

Tipler, P. \& Mosca, G. (2005). Física para la ciencia y la ingeniería, vol. II. Bogotá: Editorial Reverte. 